\title{
ROBUST BAYESIAN ESTIMATION WITH ASYMMETRIC LOSS FUNCTION
}

Abstract. The problem of robust Bayesian estimation in some models with an asymmetric loss function (LINEX) is considered. Some uncertainty about the prior is assumed by introducing two classes of priors. The most robust and conditional $\Gamma$-minimax estimators are constructed. The situations when those estimators coincide are presented.

1. Introduction and notation. In Bayesian statistical inference a statistical problem should be treated specifying a prior distribution over the parameter space. However the arbitrariness of a unique prior distribution is a permanent problem. Robust Bayesian inference deals with the problem of expressing uncertainty of the prior information using a class $\Gamma$ of priors and of measuring the range of a posterior quantity while the prior distribution $\Pi$ runs over the class $\Gamma$. This is of interest not only in calculating the range but also in constructing optimal procedures.

This paper concerns the problem of optimal estimation of an unknown real parameter. We consider two notions of optimality: conditional $\Gamma$-minimax estimators (see DasGupta and Studden (1989), Betro and Ruggeri (1992)) and stable estimators developed in Męczarski and Zieliński (1991), Boratyńska and Męczarski (1994). The first concept is connected with the problem of efficiency of the estimator with respect to the posterior risk when the priors run over $\Gamma$. The second one is connected with the problem of finding the estimator with the smallest oscillation of the posterior risk when the priors run over $\Gamma$. Sometimes those two estimators coincide (see Męczarski (1993) and Boratyńska (1997)).

2000 Mathematics Subject Classification: Primary 62C10; Secondary 62F15.

Key words and phrases: Bayes estimators, classes of priors, robust Bayesian estimation, linex loss. 
In all papers mentioned above the square loss function was considered. Being symmetric, the square loss imposes equal penalty on over- and underestimation of the same quantity.

In this paper we estimate the unknown parameter $\theta$ and consider the asymmetric loss function (LINEX)

$$
L(\theta, d)=\exp (c(d-\theta))-c(d-\theta)-1,
$$

where $c$ is a known parameter, $c>0$, and $d$ is an estimate. For motivations to use LINEX see Varian (1974), Zellner (1986), Wan, Zou, and Lee (2000) and references therein. We find the conditional $\Gamma$-minimax estimators and the stable estimators, present the conditions when those estimators coincide, in a class of models with two classes of conjugate priors given below. For the square loss, some of the presented models were considered in Boratyńska (1997). The same problem for a normal model with an unknown mean and two classes of priors was considered in Boratyńska and Drozdowicz (1999).

Let $\operatorname{Gamma}(a, b)$ be Gamma distribution on the space $\Theta=(0, \infty)$ with the Lebesgue density

$$
\pi_{a, b}(\theta)=\frac{b^{a}}{\Gamma(a)} \theta^{a-1} e^{-b \theta},
$$

where $a, b>0$ are parameters. Let

$$
R(a, b, d)=E L(\theta, d)=E e^{c(d-\theta)}+c E \theta-c d-1,
$$

where $\operatorname{Ey}(\theta)$ denotes the expected value of a function $y(\theta)$ when $\theta$ has the Gamma distribution $\operatorname{Gamma}(a, b)$. Thus

$$
R(a, b, d)=e^{c d}\left(\frac{b}{b+c}\right)^{a}+\frac{a c}{b}-c d-1 .
$$

The minimum of $R$ as a function of $d$ is attained for

$$
d_{a, b}=-\frac{1}{c} \ln E e^{-c \theta}=-\frac{1}{c} a \ln \frac{b}{b+c} .
$$

Model. Let $\left\{P_{\theta}: \theta>0\right\}$ be a one-parameter family of probability measures with densities of the form

$$
l(z, \theta)=C(z) \theta^{t(z)} e^{-s(z) \theta}, \quad z \in \mathbb{R},
$$

with respect to some $\sigma$-finite measure $\mu$ on $\mathbb{R}$, where $C, t, s$ are fixed, measurable, nonnegative functions. The popular families like Poisson, Gamma, Exponential distributions are examples. For more details see Table 1 at the end of the paper.

Let $X_{1}, \ldots, X_{n}$ be i.i.d. random variables with a distribution $P_{\theta}$. We consider the problem of estimating $\theta$ with the LINEX loss function $L(\theta, d)$. Set $X=\left(X_{1}, \ldots, X_{n}\right)$. Let $\Pi_{\alpha_{0}, \beta_{0}}=\operatorname{Gamma}\left(\alpha_{0}, \beta_{0}\right)$ be a fixed prior distribution. If $X=x$ then the posterior distribution is $\operatorname{Gamma}\left(\alpha_{0}+T, \beta_{0}+S\right)$, 
where $T=T(x, n)=\sum_{i=1}^{n} t\left(x_{i}\right)$ and $S=S(x, n)=\sum_{i=1}^{n} s\left(x_{i}\right)$. Thus the Bayes estimator is given by the formula

$$
\widehat{\theta}_{\alpha_{0}, \beta_{0}}^{\text {Bay }}=d_{\alpha_{0}+T, \beta_{0}+S}=-\frac{1}{c}\left(\alpha_{0}+T\right) \ln \frac{\beta_{0}+S}{\beta_{0}+S+c} .
$$

Now suppose that the prior distribution is not exactly specified and consider two classes of priors of $\theta$ :

$$
\Gamma_{\alpha_{0}}=\left\{\Pi_{\alpha_{0}, \beta}: \Pi_{\alpha_{0}, \beta}=\operatorname{Gamma}\left(\alpha_{0}, \beta\right), \beta \in\left[\beta_{1}, \beta_{2}\right]\right\},
$$

where $0<\beta_{1}<\beta_{2}$ are fixed and $\beta_{0} \in\left(\beta_{1}, \beta_{2}\right)$,

$$
\Gamma_{\beta_{0}}^{*}=\left\{\Pi_{\alpha, \beta_{0}}: \Pi_{\alpha, \beta_{0}}=\operatorname{Gamma}\left(\alpha, \beta_{0}\right), \alpha \in\left[\alpha_{1}, \alpha_{2}\right]\right\},
$$

where $0<\alpha_{1}<\alpha_{2}$ are fixed and $\alpha_{0} \in\left(\alpha_{1}, \alpha_{2}\right)$. The classes $\Gamma_{\alpha_{0}}$ and $\Gamma_{\beta_{0}}^{*}$ express two types of uncertainty about the elicited prior.

Let $R_{x}(\alpha, \beta, \widehat{\theta}(x))$ denote the posterior risk of an estimator $\widehat{\theta}$ when the prior is $\operatorname{Gamma}(\alpha, \beta)$. Then

$$
R_{x}(\alpha, \beta, \widehat{\theta}(x))=R(\alpha+T, \beta+S, \widehat{\theta}(x))
$$

and the ranges of the posterior risk of the estimator $\widehat{\theta}$ when the prior runs over $\Gamma_{\alpha_{0}}$ and $\Gamma_{\beta_{0}}^{*}$ are

$r_{\alpha_{0}}(\widehat{\theta}(x))=\sup _{\beta \in\left[\beta_{1}, \beta_{2}\right]} R\left(\alpha_{0}+T, \beta+S, \widehat{\theta}(x)\right)-\inf _{\beta \in\left[\beta_{1}, \beta_{2}\right]} R\left(\alpha_{0}+T, \beta+S, \widehat{\theta}(x)\right)$, $r_{\beta_{0}}^{*}(\widehat{\theta}(x))=\sup _{\alpha \in\left[\alpha_{1}, \alpha_{2}\right]} R\left(\alpha+T, \beta_{0}+S, \widehat{\theta}(x)\right)-\inf _{\alpha \in\left[\alpha_{1}, \alpha_{2}\right]} R\left(\alpha+T, \beta_{0}+S, \widehat{\theta}(x)\right)$, respectively.

The problem is to find the most stable estimators $\widehat{\theta}_{\alpha_{0}}$ and $\widehat{\theta}_{\beta_{0}}^{*}$, i.e. those satisfying

$$
\begin{array}{ll}
\forall x & \inf _{d \in \mathbb{R}} r_{\alpha_{0}}(d)=r_{\alpha_{0}}\left(\widehat{\theta}_{\alpha_{0}}(x)\right), \\
\forall x \quad \inf _{d \in \mathbb{R}} r_{\beta_{0}}^{*}(d)=r_{\beta_{0}}^{*}\left(\widehat{\theta}_{\beta_{0}}^{*}(x)\right),
\end{array}
$$

and to find the conditional $\Gamma$-minimax estimators $\widetilde{\theta}_{\alpha_{0}}$ and $\widetilde{\theta}_{\beta_{0}}^{*}$, i.e. the estimators satisfying

$$
\begin{array}{ll}
\forall x & \inf _{d \in \mathbb{R}} \sup _{\beta \in\left[\beta_{1}, \beta_{2}\right]} R_{x}\left(\alpha_{0}, \beta, d\right)=\sup _{\beta \in\left[\beta_{1}, \beta_{2}\right]} R_{x}\left(\alpha_{0}, \beta, \widetilde{\theta}_{\alpha_{0}}(x)\right), \\
\forall x & \inf _{d \in \mathbb{R}} \sup _{\alpha \in\left[\alpha_{1}, \alpha_{2}\right]} R_{x}\left(\alpha, \beta_{0}, d\right)=\sup _{\alpha \in\left[\alpha_{1}, \alpha_{2}\right]} R_{x}\left(\alpha, \beta_{0}, \widetilde{\theta}_{\beta_{0}}^{*}(x)\right) .
\end{array}
$$

2. Properties of the function $R$. Let $\left(a_{0}, b_{0}\right)$ be a fixed pair such that $a_{0}>0$ and $b_{0}>0$. Let $\left[a_{1}, a_{2}\right]$ and $\left[b_{1}, b_{2}\right]$ be two intervals such that $a_{0} \in\left(a_{1}, a_{2}\right)$ and $b_{0} \in\left(b_{1}, b_{2}\right)$ and $a_{1}, a_{2}, b_{1}, b_{2}>0$. 
LEMMA 1. (1) $R\left(a_{0}, b, \cdot\right)$ is a strictly convex function for every $b>0$.

(2) For every $d$, the minimum point $b_{\min }(d)$ of $R\left(a_{0}, \cdot d\right)$ is unique and it is a strictly decreasing function of $d$.

(3) For any $\bar{b}$ and $\bar{d}$ such that $b_{\min }(\bar{d})=\bar{b}$ we have $\forall d_{1}<d_{2} \leq \bar{d}$

$$
\frac{R\left(a_{0}, \bar{b}, d_{2}\right)-R\left(a_{0}, \bar{b}, d_{1}\right)}{d_{2}-d_{1}}<\frac{R\left(a_{0}, b_{\min }\left(d_{2}\right), d_{2}\right)-R\left(a_{0}, b_{\min }\left(d_{1}\right), d_{1}\right)}{d_{2}-d_{1}}
$$

and

$\forall d_{2}>d_{1} \geq \bar{d}$

$$
\frac{R\left(a_{0}, \bar{b}, d_{2}\right)-R\left(a_{0}, \bar{b}, d_{1}\right)}{d_{2}-d_{1}}>\frac{R\left(a_{0}, b_{\min }\left(d_{2}\right), d_{2}\right)-R\left(a_{0}, b_{\min }\left(d_{1}\right), d_{1}\right)}{d_{2}-d_{1}} .
$$

(4) $R\left(a_{0}, b_{1}, d\right)-R\left(a_{0}, b_{2}, d\right)$ is a decreasing function of $d$.

(5) The solution of the equation $R\left(a_{0}, b_{1}, d\right)=R\left(a_{0}, b_{2}, d\right)$ is

$$
d_{a_{0}}=-\frac{a_{0}}{c} \ln \frac{b_{2}}{b_{2}+c}+\frac{1}{c}\left\{\ln \left[\frac{a_{0} c}{b_{1} b_{2}}\left(b_{2}-b_{1}\right)\right]-\ln \left[1-\left(\frac{b_{1}\left(b_{2}+c\right)}{b_{2}\left(b_{1}+c\right)}\right)^{a_{0}}\right]\right\}
$$

and $d_{a_{0}}>0$ for all $b_{2}>b_{1}>0$.

(6) Let $u=\left(\frac{b_{1}\left(b_{2}+c\right)}{b_{2}\left(b_{1}+c\right)}\right)^{a_{0}}$. Then

$$
d_{a_{0}, b_{2}} \leq d_{a_{0}} \leq d_{a_{0}, b_{1}} \quad \text { iff } \quad 1-u \leq \frac{a_{0} c}{b_{1} b_{2}}\left(b_{2}-b_{1}\right) \leq \frac{1}{u}-1
$$

Proof. For (1), note that

$$
\frac{\partial^{2}}{\partial d^{2}} R\left(a_{0}, b, d\right)=c^{2} e^{c d}\left(\frac{b}{b+c}\right)^{a_{0}}>0
$$

Moreover,

$$
\frac{\partial}{\partial b} R\left(a_{0}, b, d\right)=\frac{c a_{0}}{b^{2}}\left[e^{c d}\left(\frac{b}{b+c}\right)^{a_{0}+1}-1\right]
$$

and the minimum point

$$
b_{\min }(d)=\frac{c}{e^{\frac{c d}{a_{0}+1}}-1}
$$

is a decreasing function of $d$.

To check (3) note that

$$
R\left(a_{0}, \bar{b}, d\right)=\exp \left(c d-\frac{c \bar{d} a_{0}}{a_{0}+1}\right)+a_{0} \exp \left(\frac{c \bar{d}}{a_{0}+1}\right)-a_{0}-c d-1
$$

and

$$
R\left(a_{0}, b_{\min }(d), d\right)=\left(a_{0}+1\right) \exp \left(\frac{c d}{a_{0}+1}\right)-a_{0}-c d-1 .
$$

Thus it is enough to show the inequalities 


$$
\begin{aligned}
& \forall d_{1}<d_{2} \leq \bar{d} \quad \frac{e^{\frac{-c \bar{d} a_{0}}{a_{0}+1}}\left(e^{c d_{2}}-e^{c d_{1}}\right)}{d_{2}-d_{1}}<\frac{\left(a_{0}+1\right)\left(e^{\frac{c d_{2}}{a_{0}+1}}-e^{\frac{c d_{1}}{a_{0}+1}}\right)}{d_{2}-d_{1}}, \\
& \forall d_{2}>d_{1} \geq \bar{d} \quad \frac{e^{\frac{-c \bar{d} a_{0}}{a_{0}+1}}\left(e^{c d_{2}}-e^{c d_{1}}\right)}{d_{2}-d_{1}}>\frac{\left(a_{0}+1\right)\left(e^{\frac{c d_{2}}{a_{0}+1}}-e^{\frac{c d_{1}}{a_{0}+1}}\right)}{d_{2}-d_{1}} .
\end{aligned}
$$

Consider the first inequality. It is equivalent to

$$
\forall d_{1}<d_{2} \leq \bar{d} \quad \frac{f\left(d_{2}\right)-f\left(d_{1}\right)}{d_{2}-d_{1}}<0,
$$

where

$$
f(d)=\exp \left(c d-\frac{c \bar{d} a_{0}}{a_{0}+1}\right)-\left(a_{0}+1\right) \exp \left(\frac{c d}{a_{0}+1}\right) .
$$

By the Lagrange formula it is enough to show that

$$
f^{\prime}(d)=c \exp \left(c d-\frac{c \bar{d} a_{0}}{a_{0}+1}\right)-c \exp \frac{c d}{a_{0}+1}<0
$$

for all $d<\bar{d}$. And since $g(x)=e^{x}$ is increasing, this is indeed true.

The second inequality is shown the same way.

Since

$$
\frac{\partial}{\partial d}\left(R\left(a_{0}, b_{1}, d\right)-R\left(a_{0}, b_{2}, d\right)\right)=c e^{c d}\left[\left(\frac{b_{1}}{b_{1}+c}\right)^{a_{0}}-\left(\frac{b_{2}}{b_{2}+c}\right)^{a_{0}}\right]
$$

and the function $g(b)=\left(\frac{b}{b+c}\right)^{a_{0}}$ is increasing, property (4) is true. Now easy calculations give (5) and (6).

Lemma 2. (1) $R\left(a, b_{0}, \cdot\right)$ is a strictly convex function for every $a>0$.

(2) For every $d$, the minimum point $a_{\min }(d)$ of $R\left(\cdot, b_{0}, d\right)$ is unique and it is a strictly increasing function of $d$.

(3) For any $\bar{a}$ and $\bar{d}$ such that $a_{\min }(\bar{d})=\bar{a}$ we have $\forall d_{1}<d_{2} \leq \bar{d}$

$$
\frac{R\left(\bar{a}, b_{0}, d_{2}\right)-R\left(\bar{a}, b_{0}, d_{1}\right)}{d_{2}-d_{1}}<\frac{R\left(a_{\min }\left(d_{2}\right), b_{0}, d_{2}\right)-R\left(a_{\min }\left(d_{1}\right), b_{0}, d_{1}\right)}{d_{2}-d_{1}}
$$

and

$\forall d_{2}>d_{1} \geq \bar{d}$

$$
\frac{R\left(\bar{a}, b_{0}, d_{2}\right)-R\left(\bar{a}, b_{0}, d_{1}\right)}{d_{2}-d_{1}}>\frac{R\left(a_{\min }\left(d_{2}\right), b_{0}, d_{2}\right)-R\left(a_{\min }\left(d_{1}\right), b_{0}, d_{1}\right)}{d_{2}-d_{1}} .
$$

(4) $R\left(a_{1}, b_{0}, d\right)-R\left(a_{2}, b_{0}, d\right)$ is an increasing function of $d$.

(5) The solution of the equation $R\left(a_{1}, b_{0}, d\right)=R\left(a_{2}, b_{0}, d\right)$ is equal to

$$
d_{b_{0}}^{*}=-\frac{a_{1}}{c} \ln \frac{b_{0}}{b_{0}+c}+\frac{1}{c}\left\{\ln \left[\frac{c}{b_{0}}\left(a_{2}-a_{1}\right)\right]-\ln \left[1-\left(\frac{b_{0}}{b_{0}+c}\right)^{a_{2}-a_{1}}\right]\right\}
$$

and $d_{b_{0}}^{*}>0$ for all $a_{2}>a_{1}>0$ and $b_{0}>0$. 
(6) Let $v=\left(\frac{b_{0}+c}{b_{0}}\right)^{a_{2}-a_{1}}$. Then

$$
d_{a_{1}, b_{0}} \leq d_{b_{0}}^{*} \leq d_{a_{2}, b_{0}} \quad \text { iff } \quad \frac{c}{b_{0}}\left(a_{2}-a_{1}\right) \leq v-1
$$

Proof. The proof is very similar to the proof of Lemma 1. We show only properties (3) and (6).

Let $\frac{b_{0}}{b_{0}+c}=z$. Then $z \in(0,1)$ and $b_{0}=\frac{c z}{1-z}$. We have

$$
R\left(a, b_{0}, d\right)=g(a, z, d)=e^{c d} z^{a}+\frac{a(1-z)}{z}-c d-1 .
$$

The minimum point $a_{\min }(d)$ of $g(\cdot, z, d)$ is

$$
a_{\min }(d)=\log _{z} \frac{e^{-c d}(1-z)}{-z \ln z}=\frac{-c d+\ln (1-z)-\ln \ln z^{-1}}{\ln z}-1 .
$$

We have

$$
g\left(a_{\min }(\bar{d}), z, d\right)=e^{c d}\left(-\frac{e^{-c \bar{d}}(1-z)}{z \ln z}\right)+\frac{1-z}{z} \log _{z} \frac{e^{-c \bar{d}}(1-z)}{-z \ln z}-c d-1
$$

and

$g\left(a_{\min }(d), z, d\right)=-\frac{1-z}{z \ln z}+\frac{1-z}{z}\left(\frac{-c d+\ln (1-z)-\ln \ln z^{-1}}{\ln z}-1\right)-c d-1$.

To prove (3) it is enough to show the inequalities

$$
\begin{aligned}
& \forall d_{1}<d_{2} \leq \bar{d} \quad-\frac{e^{-c \bar{d}}(1-z)}{z \ln z} \cdot \frac{e^{c d_{2}}-e^{c d_{1}}}{d_{2}-d_{1}}<-\frac{c(1-z)}{z \ln z}, \\
& \forall d_{2}>d_{1} \geq \bar{d} \quad-\frac{e^{-c \bar{d}}(1-z)}{z \ln z} \cdot \frac{e^{c d_{2}}-e^{c d_{1}}}{d_{2}-d_{1}}>-\frac{c(1-z)}{z \ln z} .
\end{aligned}
$$

They are equivalent to the inequalities

$$
\begin{array}{ll}
\forall d_{1}<d_{2} \leq \bar{d} & \frac{e^{c d_{2}}-e^{c d_{1}}}{d_{2}-d_{1}}<c e^{c \bar{d}} \\
\forall d_{2}>d_{1} \geq \bar{d} & \frac{e^{c d_{2}}-e^{c d_{1}}}{d_{2}-d_{1}}>c e^{c \bar{d}}
\end{array}
$$

which are true by the Lagrange formula for $f(d)=e^{c d}$.

To prove (6) consider the inequalities

$$
d_{a_{1}, b_{0}} \leq d_{b_{0}}^{*} \leq d_{a_{2}, b_{0}} .
$$

They are equivalent to

$$
1-\frac{1}{v} \leq \frac{c}{b_{0}}\left(a_{2}-a_{1}\right) \leq v-1
$$


where $v=\left(\frac{b_{0}+c}{b_{0}}\right)^{a_{2}-a_{1}}$. The first inequality is equivalent to

$$
\frac{1-\left(\frac{b_{0}}{b_{0}+c}\right)^{a_{2}-a_{1}}}{a_{2}-a_{1}} \leq \frac{c}{b_{0}} .
$$

Let $f(a)=\left(\frac{b_{0}}{b_{0}+c}\right)^{a_{2}-a}$. Then $f\left(a_{2}\right)=1$ and $f\left(a_{1}\right)=\left(\frac{b_{0}}{b_{0}+c}\right)^{a_{2}-a_{1}}$. We have

$$
f^{\prime}(a)=\left(\frac{b_{0}}{b_{0}+c}\right)^{a_{2}-a} \ln \left(1+\frac{c}{b_{0}}\right)
$$

and

$$
f^{\prime}(a) \leq \ln \left(1+\frac{c}{b_{0}}\right) \leq \frac{c}{b_{0}}
$$

for $a \leq a_{2}$, which completes the proof of (6).

3. The most stable and conditional $\Gamma$-minimax estimators. We use the following theorem proved by Męczarski (1993).

Theorem 1 (Męczarski (1993)). Let $\Gamma=\left\{\Pi_{\gamma}: \gamma \in\left[\gamma_{1}, \gamma_{2}\right]\right\}$ be a set of prior distributions, where $\gamma$ is a real parameter. Let $\varrho(\gamma, d)$ be a posterior risk of a decision d based on the observation $x$ when the prior is $\Pi_{\gamma}$. Assume that the function $\varrho(\gamma, d)$ satisfies the following conditions:

(1) $\varrho(\gamma, \cdot)$ is a strictly convex function for any $\gamma$;

(2) for any $d$ the minimum point $\gamma_{\min }(d)$ of $\varrho(\cdot, d)$ is unique and $\gamma_{\min }$ is a strictly monotone function of $d$;

(3) for any $\bar{\gamma}$ and $\bar{d}$ such that $\gamma_{\min }(\bar{d})=\bar{\gamma}$ we have

$$
\forall d_{1}<d_{2} \leq \bar{d} \quad \frac{\varrho\left(\bar{\gamma}, d_{2}\right)-\varrho\left(\bar{\gamma}, d_{1}\right)}{d_{2}-d_{1}}<\frac{\varrho\left(\gamma_{\min }\left(d_{2}\right), d_{2}\right)-\varrho\left(\gamma_{\min }\left(d_{1}\right), d_{1}\right)}{d_{2}-d_{1}}
$$

and

$$
\forall d_{2}>d_{1} \geq \bar{d} \quad \frac{\varrho\left(\bar{\gamma}, d_{2}\right)-\varrho\left(\bar{\gamma}, d_{1}\right)}{d_{2}-d_{1}}>\frac{\varrho\left(\gamma_{\min }\left(d_{2}\right), d_{2}\right)-\varrho\left(\gamma_{\min }\left(d_{1}\right), d_{1}\right)}{d_{2}-d_{1}} ;
$$

(4) $\varrho\left(\gamma_{1}, d\right)-\varrho\left(\gamma_{2}, d\right)$ is a monotone function of $d$.

Then

(i) if there exists $\widehat{d}$ such that

$$
\sup _{\gamma \in\left[\gamma_{1}, \gamma_{2}\right]} \varrho(\gamma, \widehat{d})=\varrho\left(\gamma_{1}, \widehat{d}\right)=\varrho\left(\gamma_{2}, \widehat{d}\right)
$$

then $\widehat{d}$ is the most stable;

(ii) let $\mathcal{L}_{\Gamma}=\left\{d: \forall x \in \mathcal{X} \exists \gamma \in\left[\gamma_{1}, \gamma_{2}\right] d(x)=d_{\gamma}^{\text {Bay }}(x)\right\}$; if $\widehat{d}$ satisfying (i) belongs to $\mathcal{L}_{\Gamma}$ then $\widehat{d}$ is conditional $\Gamma$-minimax.

We prove the following results. 
THEOREM 2. If the class of priors is equal to $\Gamma_{\alpha_{0}}$ then

$$
\begin{gathered}
\widehat{\theta}_{\alpha_{0}}=\widehat{\theta}_{\alpha_{0}, \beta_{2}}^{\text {Bay }}+\frac{1}{c}\left\{\ln \left|\frac{c\left(\alpha_{0}+T\right)\left(\beta_{2}-\beta_{1}\right)}{\left(\beta_{1}+S\right)\left(\beta_{2}+S\right)}\right|\right. \\
\left.-\ln \left|1-\left(\frac{\left(\beta_{2}+S+c\right)\left(\beta_{1}+S\right)}{\left(\beta_{2}+S\right)\left(\beta_{1}+S+c\right)}\right)^{\alpha_{0}+T}\right|\right\}
\end{gathered}
$$

and

$$
\widetilde{\theta}_{\alpha_{0}}= \begin{cases}\widehat{\theta}_{a_{0}, \beta_{1}}^{\text {Bay }} & \text { if } \frac{c\left(\alpha_{0}+T\right)\left(\beta_{2}-\beta_{1}\right)}{\left(\beta_{1}+S\right)\left(\beta_{2}+S\right)}>\frac{1}{u}-1, \\ \widehat{\theta}_{\alpha_{0}} & \text { if } 1-u \leq \frac{c\left(\alpha_{0}+T\right)\left(\beta_{2}-\beta_{1}\right)}{\left(\beta_{1}+S\right)\left(\beta_{2}+S\right)} \leq \frac{1}{u}-1, \\ \widehat{\theta}_{\alpha_{0}, \beta_{2}}^{\text {Bay }} & \text { if } \frac{c\left(\alpha_{0}+T\right)\left(\beta_{2}-\beta_{1}\right)}{\left(\beta_{1}+S\right)\left(\beta_{2}+S\right)}<1-u,\end{cases}
$$

where

$$
u=\left[\frac{\left(\beta_{1}+S\right)\left(\beta_{2}+S+c\right)}{\left(\beta_{2}+S\right)\left(\beta_{1}+S+c\right)}\right]^{\alpha_{0}+T} .
$$

The most stable estimator in the class

$$
\mathcal{L}_{\alpha_{0}}=\left\{\widehat{\theta}: \forall x \exists \beta \in\left[\beta_{1}, \beta_{2}\right] \widehat{\theta}(x)=\widehat{\theta}_{\alpha_{0}, \beta}^{\text {Bay }}(x)\right\}
$$

is equal to the conditional $\Gamma$-minimax estimator $\widetilde{\theta}_{\alpha_{0}}$.

Proof. The posterior risk of an estimator $\widehat{\theta}$ for $X=x$ and the prior $\Pi_{\alpha_{0}, \beta}$ is

$$
R_{x}\left(\alpha_{0}, \beta, \widehat{\theta}(x)\right)=R\left(a_{0}, b, \widehat{\theta}(x)\right),
$$

where $a_{0}=\alpha_{0}+T, b=\beta+S$ and $b$ is an increasing, linear function of $\beta$. Let $b_{1}=\beta_{1}+S$ and $b_{2}=\beta_{2}+S$. By Lemma 1 conditions (1)-(4) of Theorem 1 hold for the function $\varrho(b, d)=R\left(a_{0}, b, d\right)$. Thus by Lemma 1(5) we obtain the most stable estimator.

To find the conditional $\Gamma$-minimax estimator we use Lemma 1(4)-(6) and note that

$$
\sup _{b \in\left[b_{1}, b_{2}\right]} \varrho(b, d)= \begin{cases}\varrho\left(b_{1}, d\right) & \text { for } d \leq \widehat{\theta}_{\alpha_{0}}(x), \\ \varrho\left(b_{2}, d\right) & \text { for } d \geq \widehat{\theta}_{\alpha_{0}}(x) .\end{cases}
$$

Thus if $\widehat{\theta}_{\alpha_{0}}>\widehat{\theta}_{\alpha_{0}, \beta_{1}}^{\text {Bay }}$ for $X=x$ then also $\widehat{\theta}_{\alpha_{0}}>\widehat{\theta}_{\alpha_{0}, \beta_{2}}^{\text {Bay }}$, the oscillation $r_{\alpha_{0}}(d)$ is a decreasing function of $d$ for $d<\widehat{\theta}_{\alpha_{0}}(x)$ and

$$
\inf _{d \geq \widehat{\theta}_{\alpha_{0}}(x)} \sup _{b \in\left[b_{1}, b_{2}\right]} \varrho(b, d)=\varrho\left(b_{2}, \widehat{\theta}_{\alpha_{0}}(x)\right) .
$$

But

$$
\varrho\left(b_{2}, \widehat{\theta}_{\alpha_{0}}(x)\right)=\varrho\left(b_{1}, \widehat{\theta}_{\alpha_{0}}(x)\right) \geq \inf _{d \leq \widehat{\theta}_{\alpha_{0}}(x)} \sup _{b \in\left[b_{1}, b_{2}\right]} \varrho(b, d)=\varrho\left(b_{1}, \widehat{\theta}_{\alpha_{0}, \beta_{1}}^{\text {Bay }}(x)\right) .
$$

Similarly we consider the case $\widehat{\theta}_{\alpha_{0}}<\widehat{\theta}_{\alpha_{0}, \beta_{2}}^{\text {Bay }}$ and complete the proof. 
TheOrem 3. If the class of priors is equal to $\Gamma_{\beta_{0}}^{*}$ then

$$
\widehat{\theta}_{\beta_{0}}^{*}=\widehat{\theta}_{\alpha_{1}, \beta_{0}}^{\mathrm{Bay}}+\frac{1}{c}\left\{\ln \left|\frac{c\left(\alpha_{2}-\alpha_{1}\right)}{\beta_{0}+S}\right|-\ln \left|1-\left(\frac{\beta_{0}+S}{\beta_{0}+S+c}\right)^{\alpha_{2}-\alpha_{1}}\right|\right\}
$$

and

$$
\widetilde{\theta}_{\beta_{0}}^{*}= \begin{cases}\widehat{\theta}_{\alpha_{2}, \beta_{0}}^{\mathrm{Bay}} & \text { if } \frac{c\left(\alpha_{2}-\alpha_{1}\right)}{\beta_{0}+S}>v-1, \\ \widehat{\theta}_{\beta_{0}}^{*} & \text { if } \frac{c\left(\alpha_{2}-\alpha_{1}\right)}{\beta_{0}+S} \leq v-1,\end{cases}
$$

where

$$
v=\left(\frac{\beta_{0}+S+c}{\beta_{0}+S}\right)^{\alpha_{2}-\alpha_{1}} .
$$

The most stable estimator in the class

$$
\mathcal{L}_{\beta_{0}}^{*}=\left\{\widehat{\theta}: \forall x \exists \alpha \in\left[\alpha_{1}, \alpha_{2}\right] \widehat{\theta}(x)=\widehat{\theta}_{\alpha, \beta_{0}}^{\text {Bay }}(x)\right\}
$$

is equal to the conditional $\Gamma$-minimax estimator $\widetilde{\theta}_{\beta_{0}}^{*}$.

Proof. The posterior risk of an estimator $\widehat{\theta}$ for $X=x$ and the prior $\Pi_{\alpha, \beta_{0}}$ is

$$
R_{x}\left(\alpha, \beta_{0}, \widehat{\theta}(x)\right)=R\left(a, b_{0}, \widehat{\theta}(x)\right),
$$

where $b_{0}=\beta_{0}+S, a=\alpha+T$ and $a$ is an increasing, linear function of $\alpha$. Let $a_{1}=\alpha_{1}+T$ and $a_{2}=\alpha_{2}+T$. Lemma 2 shows that conditions (1)-(4) of Theorem 1 are satisfied for the function $\varrho(a, d)=R\left(a, b_{0}, d\right)$ and

$$
\sup _{a \in\left[a_{1}, a_{2}\right]} \varrho(a, d)= \begin{cases}\varrho\left(a_{2}, d\right) & \text { for } d \leq \widehat{\theta}_{\beta_{0}}^{*}(x), \\ \varrho\left(a_{1}, d\right) & \text { for } d \geq \widehat{\theta}_{\beta_{0}}^{*}(x) .\end{cases}
$$

Analysis similar to that in the proof of Theorem 2 completes the proof.

4. Examples and remarks. Table 1 presents some examples of families $\left\{P_{\theta}: \theta \in \Theta\right\}$ and functions $T(x, n)$ and $S(x, n)$.

REMARK 1. If there exists a constant $B_{1}>0$ such that $\beta_{1}+S>B_{1}$ for all $x$, then Theorem 2 is also true for $c \in\left(-B_{1}, 0\right)$.

REMARK 2. If there exists a constant $B_{2}>0$ such that $\beta_{0}+S>B_{2}$ for all $x$, then Theorem 3 is also true for $c \in\left(-B_{2}, 0\right)$. 
Table 1

\begin{tabular}{ccc}
\hline Distribution & $T(x, n)$ & $S(x, n)$ \\
\hline Poisson & $\sum_{i=1}^{n} x_{i}$ & $n$ \\
$P(\theta)$ & $n$ & $\sum_{i=1}^{n} x_{i}$ \\
Exponential & $n$ & $\sum_{i=1}^{n} x_{i}$ \\
$E(\theta)$ & $n p$ & $\frac{1}{2} \sum_{i=1}^{n}\left(x_{i}-\mu\right)^{2}$ \\
Gamma $(p, \theta)$ & & \\
$p$ known & & $\sum_{i=1}^{n} \ln \frac{\lambda+x_{i}}{\lambda}$ \\
Normal $N\left(\mu, \frac{1}{\theta}\right)$ & $\frac{1}{2} n$ & \\
$\mu$ known & & \\
Pareto $(\lambda, \theta)$ & $n$ & \\
$\lambda$ known & &
\end{tabular}

\section{References}

B. Betro and F. Ruggeri (1992), Conditional $\Gamma$-minimax actions under convex losses, Comm. Statist. Theory Methods 21, 1051-1066.

A. Boratyńska (1997), Stability of Bayesian inference in exponential families, Statist. Probab. Lett. 36, 173-178.

A. Boratyńska and M. Drozdowicz (1999), Robust Bayesian estimation in a normal model with asymmetric loss function, Appl. Math. (Warsaw) 26, 85-92.

A. Boratyńska and M. Męczarski (1994), Robust Bayesian estimation in the one-dimensional normal model, Statistics Decisions 12, 221-230.

A. DasGupta and W. J. Studden (1989), Frequentist behavior of robust Bayes estimates of normal means, ibid. 7, 333-361.

M. Męczarski (1993), Stability and conditional $\Gamma$-minimaxity in Bayesian inference, Appl. Math. (Warsaw) 22, 117-122.

M. Męczarski and R. Zieliński (1991), Stability of the Bayesian estimator of the Poisson mean under the inexactly specified gamma prior, Statist. Probab. Lett. 12, 329-333.

H. R. Varian (1974), A Bayesian approach to real estate assessment, in: Studies in Bayesian Econometrics and Statistics, North-Holland, 195-208.

A. Wan, G. Zou and A. Lee (2000), Minimax and $\Gamma$-minimax estimation for the Poisson distribution under LINEX loss when the parameter space is restricted, Statist. Probab. Lett. 50, 23-32.

A. Zellner (1986), Bayesian estimation and prediction using asymmetric loss functions, J. Amer. Statist. Assoc. 81, 446-451.

Institute of Econometrics

Warsaw School of Economics

Al. Niepodległości 162

02-554 Warszawa, Poland

E-mail: aborata@sgh.waw.pl 